\title{
AN ASYMPTOTIC FORMULA IN ADELE DIOPHANTINE APPROXIMATIONS
}

BY

\author{
MELVIN M. SWEET
}

\begin{abstract}
In this paper an asymptotic formula is found for the number of solutions of a system of linear Diophantine inequalities defined over the ring of adeles of an algebraic number field. The theorem proved is a generalization of results of S. Lang and W. Adams.
\end{abstract}

1. Introduction. Serge Lang [ 5 ] defines a number to have type $\leq g$ if $g$ is a positive increasing function for which $|q b-p| \geq 1 / q g(q)$ for all $q$ sufficiently large. Lang then shows that the number $\lambda(N, b)$ of solutions of $|q b-p| \leq \psi(q)$ with $q \leq N$ is asymptotic to $S_{N}=\sum_{q=1}^{N} 2 \psi(q)$ if $b$ has type $\leq g$ and $\psi$ decreases so slowly that $\psi(q) q g(q)^{-1}$ increases to infinity with $q$. W. Adams [1] has extended this result of Lang to the simultaneous approximation of real numbers by rationals. I have also shown in [8] how these results may be extended to linear forms. The purpose of this paper is to show that the Lang-Adams theorem holds for the approximation of linear forms in the ring of adeles over a number field $k$. A p-adic theorem, as well as some of the results in [8], could be stated as corollaries to the theorem proved here. The theorem proved is probably not the best possible such theorem. This is suggested by a metric example I will give later.

Diophantine approximations over the adeles have previously been considered by David Cantor in [2]. In his paper Cantor shows adele analogues of some of the basic theorems. To some extent, I have followed Cantor in notation and setting up the problem in the ring of adeles.

I wish to thank Professor W. Adams for his help and encouragement in my work.

2. Notation. We use $k$ to denote an algebraic number field of degree $n$ with ring of integers $D$. Let $P$ be the set of all primes of $k$. We write $P_{\infty}$ for the set of all infinite primes, and $P_{0}$ for the set of all finite primes. When $P_{0}$ and $P_{\infty}$ are used as subscripts, we will replace them by 0 and $\infty$ respectively. For $p \in P$, we let $k_{p} \supseteq k$ denote the completion of $k$ with respect to $k$

We may assume $P_{0}$ is the set of all prime ideals of $D_{0}$. For $\not \in P_{0}, x \in k$, let $\nu=\nu_{p}(x)$ be the p-order of $x$. We normalize the absolute value ||$_{p}$ associated with $p$ so that $|x|_{p}=N p^{-\nu}$, where $N p$ is the norm of the ideal $p$.

Presented to the Society, January 18, 1972 under the title Counting solutions of adèle Diophantine approximations; received by the editors May 21, 1973.

AMS (MOS) subject classifications (1970). Primary 10F30, 10F 45. 
Let $x \rightarrow x^{(i)}, i=1, \cdots, n$, be the embeddings of $k$ into $\mathrm{C}$, the complex numbers. We arrange the notation so that the first $R_{1}$ embeddings map into the real numbers $R$ and the remaining maps consist of $R_{2}$ pairs of complex conjugate mappings listed so that

$$
x^{\left(R_{1}+R_{2}+i\right)}=x^{\overline{\left(R_{1}+i\right)}} \text { for } i=1, \ldots, R_{2} .
$$

The infinite primes of $k$ can be identified with the first $R=R_{1}+R_{2}$ of these mappings. We use || to stand for the ordinary absolute value on $C$. If $\mathfrak{F}$ is the infinite prime corresponding to $x \rightarrow x^{(i)}$, then we set $|x|_{p}=\left|x^{(i)}\right|$ if $k^{(i)}$ is real, otherwise we set $|x|_{p}=\left|x^{(i)}\right|^{2}$. The infinite prime $\mathfrak{p}$ is called real when $k^{(i)} \subseteq$ $\mathbf{R}$ and complex otherwise. If $p$ is real, then $k_{p}=\mathbf{R}$ and we will often identify $k$ with a subfield of $\mathbf{R}$ by means of $x \rightarrow x^{(i)}$. A similar statement can be made when $\mathfrak{p}$ is complex, in which case $k_{\mathfrak{p}}=\mathrm{C}$. Hence, if we write ||$_{\mathfrak{p}}$ for the extension of ||$_{p}$ to $k_{p}$, we may think of ||$_{p}$ as the ordinary absolute value when $k_{p}=\mathbf{R}$ and the square of the ordinary absolute value when $k_{p}=\mathbf{C}$.

For $p \in P_{0}$, the set $D_{p}$ of all $x$ in $k_{p}$ for which $|x|_{p} \leq 1$ is the ring of $p$ adic integers of $k_{p}$. For $p \in P_{\infty}$, we set $D_{p}=k_{p}$.

Let $S$ be any subset of $P$. Consider the product $\Pi k_{p}$ over all $p \in S$, with componentwise algebraic operations. For any $a$ in this product we use $a_{p}$ to stand for the pth component of $a$. We define the ring $k_{S}$ of $S$-adeles to be the subset of this product consisting of all $a$ with $a_{p} \in D_{p}$ for all but a finite number of $p$. Note that this is not the ring usually referred to as the $S$-adele ring. We embed $k$ in $k_{S}$ by identifying $a \in k$ with the element in $k_{S}$, also denoted by $a$, for which $a_{p}=a \in k$ for all $p \in S$. We let $S_{\infty}=S \cap P_{\infty}$ and $S_{0}=S \cap P_{0}$. Then we can write $k_{S}=k_{S_{\infty}} \times k_{S_{0}}$. For $a \in k_{S}$ we write $a^{\infty}$ for the $k_{S_{\infty}}$ component of $a$, and we write $a^{b}$ for the $k_{s_{0}}$ component of $a$.

We denote the multiplicative group of units of $k_{S}$ by $k_{S}^{*}$, and call this the group of $S$-ideles. Clearly, $a \in k_{S}$ is an idele if and only if $a_{p}$ is nonzero for all $p$ in $S$ and $\left|a_{p}\right|_{p}=1$ for all but a finite number of $p \in S$.

We extend ||$_{p}$ to $k_{S}$ by defining $|a|_{p}=\left|a_{p}\right|_{p}$ for $a$ in $k_{S}$. For $T \subseteq S$ and $a \in k_{S}$, put $|a|_{T}=\Pi_{p \in T}|a|_{p}$, if this product converges; and otherwise set $|a|_{T}$ $=0$. So, if $a \in k_{S}^{*}$, then $|a|_{T} \neq 0$. For $a, b \in k_{S}$, write $a \leq b$ if $|a|_{p} \leq|b|_{p}$ for all $p \in S$, and write $a<b$ if $a \leq b$ and $|a|_{p}<|b|_{p}$ for all infinite primes in $S$. If $S \supseteq P_{\infty}$ and $x=\left(x_{1}, \ldots, x_{m}\right) \in k_{S}^{m}$ we write $|x|=\max \left|x_{i}\right|_{p}^{1 / n_{p}}$ where $n_{p}$ is the local degree of $p$ and the max is taken over all $p \in P_{\infty}$ and all $i$ satisfying $1 \leq$ $i \leq m$.

We topologize $k_{S}$ in the usual way by requiring that the sets $\left\{x \in k_{S}: x-b\right.$ $\leq a\}, a \in k_{S}^{*}$, form a neighborhood basis at $b$ in $k_{S}$. This makes $k_{S}$ into a locally compact additive topological group. 
It is well known that $D$ is a discrete subset of $k_{\infty}$ and $k_{\infty} / D$ is compact. If $S \subseteq P_{0}$, by the strong approximation theorem, $k$ is dense in $k_{S^{\circ}}$

We now define some measures, all of which will be denoted by $\mu$ when there is no ambiguity. Let $\mu_{p}$ be the Haar measure on $k_{p}$ normalized so that $\mu_{p}\left(D_{p}\right)=$ 1 when $p \in P_{0}$, and so that $\mu_{p}$ is ordinary Lebesgue measure when $\not \in P_{\infty}$. The Haar measure $\mu_{S}$ on $k_{S}$ is normalized by requiring that this measure agree with the product measure on

$$
k_{S}(T)=\prod_{p \in T} k_{p} \times \prod_{p \in S-T} D_{p}
$$

where $T$ is any finite subset of $S$. So

$$
\mu_{S}\left\{x \in k_{S}: x \leq a\right\}=2{ }^{R} 1_{\pi}{ }^{R}|a|_{S} .
$$

Whenever we talk about a measure on $k_{S}^{m}$ we mean the product measure $\mu_{S}^{m}$. If $G$ is a discrete subgroup of $k_{S}^{m}$ we will always take the counting measure. Furthermore, if $k_{S}^{m} / G$ is compact we normalize the measure $\mu$ on this group so that the measure of the group is just the $\mu_{S}^{m}$ measure of any measurable set of representatives in $k_{S}^{m}$ of the cosets of $G$. So $\mu\left(k_{\infty}^{m} / D^{m}\right)=2^{-m R_{2}}|d|^{m / 2}$ where $d$ will always stand for the discriminant of $k$.

If $\sigma$ is a topological automorphism of $k_{S}^{m}$ the modulus of $\sigma$ is defined by $\bmod \sigma=\mu(\sigma X) / \mu(X)$ where $X$ is any measurable set in $k_{s}$. If $\sigma$ is a $k_{S}$ module automorphism of $k_{S}^{m}$ with determinant $\operatorname{det} \sigma$, then $\bmod \sigma=|\operatorname{det} \sigma|_{S^{*}}$

3. Statement of the theorem. Let $L$ be the system

$$
L_{i}(x)=\sum_{j=1}^{s} a_{i j} x_{j}, \quad i=1, \ldots, r,
$$

of linear forms with coefficients in $k_{s}$. Set $m=r+s$. We will suppose $z=$ $\left(z_{1}, \cdots, z_{m}\right), x=\left(x_{1}, \cdots, x_{s}\right)$, and $y=\left(y_{1}, \ldots, y_{r}\right)$ are related by $z=(x, y)$. Suppose $A_{p}$ is the pth component of the coefficient matrix of the system

$$
L_{i}^{0}(z)=\sum_{j=1}^{s} a_{i j}^{0} z_{j}-z_{i+s}, \quad 1 \leq i \leq r .
$$

Write $\delta_{\mathfrak{p}}$ for the determinant of the $r \times r$ submatrix of $A_{\mathfrak{p}}$ with the $p$-adic absolute value of its determinant maximal. We define $\delta=\delta(L)=\left(\delta_{p}\right) \in k_{S^{\circ}}$. For simplicity, we will assume that $S \supseteq P_{\infty}$, except when we specifically state otherwise.

We let $\psi$ be a mapping from the positive reals $\mathbf{R}_{+}$to $k_{S}^{*}$. We would like to count the number $\lambda(N)$ of solutions $x \in D^{s}, y \in D^{r}$ of

$$
L_{i}(x)-y_{i}<\psi(\mid \sqrt{x}), \quad 1 \leq i \leq r,
$$

$\lceil\mid \leq N$. 
We will show how to do this when $|\psi(t)|_{S}$ does not decrease too fast. Note, there are only finitely many $x \in D^{s}$ with $|x| N$, because $|x| \leq N$ defines a bounded region in $k_{\infty}^{s}=\mathbf{R}^{s n}$ which therefore contains a finite number of points of the lattice $D^{s}$. Also, in the same way the number of $y$ corresponding to a given $x$ in (2) is finite. In fact, if $|\psi(t)|_{\infty}<2^{-n}$, then $y$ is uniquely determined; for, if $y^{\prime}$ and $y^{\prime \prime}$ both correspond to the same $x$, then

$$
y_{i}=y_{i}^{\prime}-y_{i}^{\prime \prime} \leq H \max \left\{L_{i}(x)-y_{i}^{\prime}, L_{i}(x)-y_{i}^{\prime \prime}\right\} \leq H \psi(|x|),
$$

so

$$
\mid \text { Norm } y_{i}|=| y_{i}^{\prime}-\left.y_{i}^{\prime \prime}\right|_{\infty} \leq|H \psi(|冈|)|_{\infty}<1
$$

and thus $y=0$.

We use $M$ to denote the transpose system

$$
M_{j}(y)=\sum_{i=1}^{r} a_{i j} y_{i}, \quad 1 \leq j \leq s .
$$

Let $g: \mathbf{R}_{+} \rightarrow \mathbf{R}_{+}$be an increasing function. We say $L$ has type $\leq g$ if

$$
\max _{j}\left|M_{j}(y)-x_{j}\right| s \leq g(\mid z)^{-1}|z|^{-r n / s}
$$

has only finitely many solutions $z=(x, y) \in D^{m}$. The motivation for the righthand side of ( 3 ) is the following version of Dirichlet's theorem.

Proposition. If $S \supseteq P_{\infty}$ then there are infinitely many $z=(x, y) \in D^{m}$ sucb that

$$
\left|L_{i}(x)-y_{i}\right|_{s} \leq c|\bar{z}|^{-s n / r}, \quad 1 \leq i \leq r
$$

If $S \subseteq P_{0}$ then there are infinitely many $z \in \mathcal{D}^{m}$ such that

$$
\left|L_{i}(x)-y_{i}\right|_{s} \leq\left. c \sqrt{z}\right|^{-m n / r}, \quad 1 \leq i \leq r
$$

Here $c$ is some constant depending on $k$ and $L$.

A proof of a slightly different version of this adele theorem may be found in [2, Theorem 2.3].

We prove the following theorem.

Theorem. Assume the following:

(i) $L$ bas type $\leq g$.

(ii) $\psi(t)$ is decreasing. 
(iii) $F(t)^{n(r+2 s)}=|\psi(t)|_{s}^{r} t^{s n} g\left(t^{s / \eta-s}\right.$ increases to $\infty$.

(iv) $\psi^{0}(t) \leq 1$, i.e., $|\psi(t)|_{p} \leq 1$ for all finite primes $b \in S$.

(v) $|\psi(t)|_{p_{1}}|\psi(t)|_{p_{2}}^{-1} \leq C$ for all pairs of infinite primes $p_{1}, p_{2}$, where $C$ is a constant independent of $t$.

Then the number $\lambda(N)$ of solutions of $(2)$ is

$$
\lambda(N)=\gamma \int_{1}^{N} t^{s n-1}|\psi(t)|_{S}^{r} d t+O\left(\int_{1}^{N} \frac{t^{s n-1}|\psi(t)|_{S}^{r}}{F(t)} d t\right)
$$

with $\gamma=n s 2^{R m} \pi^{m R}|\delta(L)|_{s_{0}}^{-1}|d|^{-m / 2}$.

Remark. If we specialize the type theorem to the case $k=Q, S=P_{\infty}$, we get the homogeneous version of the theorem in [8].

Remark. If we assume $S \subseteq P_{0}$, delete condition ( $\left.\nabla\right)$, and replace the righthand side in condition (iii) by $|\psi(t)|_{s}^{r} t^{m n} g\left(t^{s / \eta}\right)^{-s}$, then we can show, by making only minor changes in the proof of the above theorem, that the number of solutions of (2) and $|y| \leq N$ satisfies

$$
\lambda(N) \sim \gamma \int_{1}^{N} t^{m n-1}|\psi(t)|_{s}^{r} d t
$$

for some constant $\gamma$. This specializes to a $p$-adic theorem when $k=Q$. A similar result may be proved when $S$ includes some but not all primes of $P_{\infty}$.

In $\$ 4$ I develop some results from the geometry of numbers which I will need when I prove the above theorem in $\$ 5$. In $\$ 6$ I will show how a metric result follows from this theorem.

4. The geometry of numbers over $k$. We call $\Lambda$ an $m$-dimensional D-lattice if $\Lambda$ is a discrete $D$ submodule of $k_{\infty}^{m}$ and $k_{\infty}^{m} / \Lambda$ is compact; this last condition is the same as requiring that $\Lambda$ contain $m k$-independent elements. We call $\mu\left(k_{\infty}^{m} / \Lambda\right)$ the determinant of $\Lambda$ and denote this by det $\Lambda$. Note that $D^{m}$ is a lattice with det $=2^{-m R_{2}}|d|^{m / 2}$. From our identification of $k_{\infty}$ with $\mathbf{R}^{R_{1}} \times \mathbf{C}^{R_{2}} \cong$ $\mathbf{R}^{n}$, it is clear that an D-lattice is just an ordinary $R^{n m}$ lattice with the same determinant. Note that not every lattice in $\mathbf{R}^{n m}$ is an D-lattice.

If $a$ is an ideal of $k$, we let $a \Lambda$ be the set of all sums $\Sigma a_{i} x_{i}$ with $a_{i}$ in $a$ and $x_{i}$ in $\Lambda$. It has been shown by K. Rogers and H. P. F. Swinnerton-Dyer $[7$, Theorem 1] that

Proposition 1. If $\Lambda$ is an $\mathrm{D}$-lattice in $k_{\infty}^{m}$, there exist $m k_{\infty}$ independent points $P_{1}, \ldots, P_{m}$ in $\Lambda$ and an ideal $b \supseteq D$ in $k$ such that

$$
\Lambda=D P_{1}+\cdots+D P_{m-1}+b P_{m}
$$

where the ideal class of $\mathfrak{b}$ depends only on $\Lambda$. 
We may now state the following:

Proposition 2. a $\mathrm{A}$ is an D-lattice with det $a \Lambda=N a^{m} \operatorname{det} \Lambda$.

Proof. The first assertion follows from the expression

$$
a \Lambda=a P_{1}+\cdots+a P_{m-1}+a b P_{m} .
$$

To prove the second assertion we may suppose $a$ is integral. Then $a \Lambda \subseteq \Lambda$ and

$$
\begin{aligned}
\Lambda / a \Lambda & =\frac{D P_{1}+\cdots+D P_{m-1}+b P_{m}}{a P_{1}+\cdots+a P_{m-1}+a b P_{m}} \\
& \cong(D / a)^{m-1} \times b / a b \cong(D / a)^{m}
\end{aligned}
$$

so the order of $\Lambda / a \Lambda$ is $(N a)^{m}$. The proposition now follows.

For $x=\left(x_{1}, \cdots, x_{m}\right), y=\left(y_{1}, \cdots, y_{m}\right)$ we denote the dot product by $x \cdot y$ $=\Sigma_{i} x_{i} y_{i}$ Also, let $\operatorname{Tr}$ denote the trace function extended to $k_{\infty^{\circ}}$ We define

$$
\begin{aligned}
\Lambda^{-1} & =\left\{x \in k_{\infty}^{m}: x \cdot y \in D \text { for all } y \in \Lambda\right\}, \\
\Lambda^{*} & =\left\{x \in k_{\infty}^{m}: \operatorname{Tr}(x \cdot y) \in \mathbf{Z} \text { for all } y \in \Lambda\right\} .
\end{aligned}
$$

It is straightforward to show

Proposition 3. $\Lambda^{*}=\mathscr{D}^{-1} \Lambda^{-1}$, where $\mathfrak{D}$ is the different of $k$, i.e. $\mathfrak{D}^{-1}$ is the fractional ideal consisting of all $x \in k$ such that $\operatorname{Tr}(a x) \in \mathbf{Z}$ for all $a \in D$.

If $P_{1}, \ldots, P_{m}$ are the independent points in Proposition 1, we can find points $P_{1}^{\prime}, \ldots, P_{m}^{m}$ such that

$$
P_{i} \cdot P_{j}^{\prime}= \begin{cases}1, & i=j, \\ 0, & \text { otherwise. }\end{cases}
$$

So, $\Lambda^{-1}=D P_{1}^{\prime}+\cdots+D P_{m-1}^{\prime}+b^{-1} P_{m}^{\prime}$, and hence $\Lambda^{-1}$, and therefore, also, $\Lambda^{*}$, is an D-lattice. We call $\Lambda^{*}$ the polar lattice of $\Lambda$. This is just the ordinary polar lattice in $R^{n m}$ with respect to the bilinear form $\langle x, y\rangle=\operatorname{Tr}(x \cdot y)$.

We now give some examples of D-lattices we will need latter.

Example 1. Let $L$ be the independent system $L_{i}(z)=\sum_{j=1}^{m} a_{i j} z_{j}, 1 \leq i \leq m$, with $a_{i j} \in k_{\infty}$. The coefficient matrix $A$ of this system has determinant in $k_{\infty}^{*}$. So, $L$ determines an automorphism $L: z \rightarrow L(z)=z A$ of $k_{\infty}^{m}$ with $\bmod L=$ $|\operatorname{det} A|_{\infty^{\bullet}}$ If $\Lambda$ is an D-lattice, then so is $L(\Lambda)$. It is clear that

$$
\operatorname{det} L(\Lambda)=\bmod L \operatorname{det} \Lambda=|\operatorname{det} A|_{\infty} \operatorname{det} \Lambda .
$$

Now, let $M$ be the system with coefficient matrix ${ }^{t} A^{-1}$ (the $t$ stands for transpose). Then 


$$
L(z) \cdot M(w)=(z A) \cdot\left(w^{t} A^{-1}\right)=z A A^{-1}\left({ }^{t} w\right)=z \cdot w .
$$

Hence $L(\Lambda)^{-1}=M\left(\Lambda^{-1}\right)$ and therefore also

$$
L(\Lambda)^{*}=\mathscr{D}^{-1} L(\Lambda)^{-1}=\mathscr{D}^{-1} M\left(\Lambda^{-1}\right)=M\left(D^{-1} \Lambda^{-1}\right)=M\left(\Lambda^{*}\right) .
$$

Example 2. Assume $S \subseteq P_{0}$, and let $L$ be the system of independent linear forms $L_{i}(z)=\sum_{j=1}^{m} a_{i j} z_{j}, 1 \leq i \leq r \leq m$, with coefficients $a_{i j} \in k_{S}$. Let $\epsilon$ be an idele $\leq 1$ in $k_{S}$ and define

$$
\Lambda=\Lambda_{L, \epsilon}=\left\{z \in 0^{m} \subseteq k_{\infty}^{m}: L_{i}(z) \leq \epsilon, 1 \leq i \leq r\right\} .
$$

Since all $p \in S$ are nonarchimedean the set $\Lambda_{L, \epsilon}$ is an 0 -module. The set is discrete because $\Lambda_{L, \epsilon} \subseteq D^{m}$. Also, it contains the $m k_{\infty}$-independent elements $a e_{i}$ where $a$ is an appropriately chosen element of $D$ and $e_{i}$ is the $m$-tuple with 1 in the $i$ th position and 0 elsewhere. Hence $\Lambda_{L, \epsilon}$ is an D-lattice.

We compute the determinant of $\Lambda_{L, \epsilon^{*}}$ Let $A$ be the $r \times m$ coefficient matrix of the system $L$ and let $A_{\mathfrak{p}}$ be the pth component of this matrix. Write $\delta_{\mathfrak{p}}$ for the determinant of the $r \times r$ submatrix of $A_{p}$ with the $p$-adic absolute value of its determinant maximal. Also, write $\delta_{p}^{\prime}$ for the determinant of the submatrix of $A_{p}$ with the $\mathfrak{p}$-adic absolute value of its determinant maximum; this last submatrix may be of any size $i \times i$ with $0 \leq i \leq r$, and by convention we take the determinant of a $0 \times 0$ matrix to be 1 . We define $\delta=\left(\delta_{p}\right) \in k_{s}, \delta^{\prime}=\left(\delta_{p}^{\prime}\right) \in k_{S^{\circ}}$.

Proposition 4. If $\delta, \delta^{\prime}$ are ideles and $\epsilon \leq \delta / \delta^{\prime}$, then

$$
\operatorname{det} \Lambda_{L, \epsilon}=2^{-m R}{ }^{-m}|d|^{m / 2}\left|\epsilon^{-r} \delta\right|_{S}
$$

Proof. It suffices to prove the order of $D^{m} / \Lambda$ is $\left|\epsilon^{-r} \delta\right|_{S}$. Set

$$
\begin{aligned}
& E=\left\{z \in k_{S}^{m}: z_{i} \leq 1,1 \leq i \leq m\right\}, \\
& E^{\prime}=\left\{z \in k_{S}^{m}: L_{i}(z) \leq \epsilon, z_{j} \leq 1,1 \leq i \leq r, 1 \leq j \leq m\right\} .
\end{aligned}
$$

Since all $\wp$ in $S$ are nonarchimedean, $E$ and $E^{\prime}$ are groups with $E^{\prime} \subseteq E$. Because $D^{m}$ is dense in $E$, each coset of $E^{\prime}$ in $E$ contains an element of $D^{m}$ and therefore the injection $D^{m} \rightarrow E$ induces an isomorphism $0^{m} / \Lambda \cong E / E^{\prime}$. Thus, we need to find the order $\#\left(E / E^{\prime}\right)$ of $E / E^{\prime}$. But $\mu(E)=1$. So $\#\left(E / E^{\prime}\right)=\mu\left(E^{\prime}\right)^{-1}$, and therefore it suffices to prove $\mu\left(E^{\prime}\right)=\left|\epsilon^{r} \delta^{-1}\right|_{S}$.

Consider the inequalities

$$
\epsilon^{-1} L_{i}(z) \leq 1, \quad 1 \leq i \leq r, \quad z_{j} \leq 1, \quad 1 \leq j \leq m .
$$


Let $B$ be the coefficient matrix of the left-hand side of (5), and let $B_{p}$ be the pth component of $B$. Let $C_{p}$ denote the $m \times m$ submatrix of $B_{p}$ with the $p$-adic absolute value of its determinant maximum. Clearly, det $C_{p}=\epsilon_{p}^{-j}$ det $D_{p}$ where $D_{p}$ is a $j \times j$ submatrix of $A_{p}$. I claim that $j=r$, and therefore, clearly, det $D_{p}$ $=\delta_{p}$. Suppose that $j<r$. The submatrix of $A_{p}$ with determinant $\delta_{p}$ yields a submatrix of $B_{p}$ with determinant $\epsilon_{p}^{-r} \delta_{p}$; so $\left|\epsilon^{-r} \delta\right|_{p}<\mid \epsilon_{p}^{-j}$ det $\left.D_{p}\right|_{p}$ and therefore

$$
|\epsilon|_{p} \geq|\epsilon|_{p}^{r-j}>\left|\delta_{p} / \operatorname{det} D_{p}\right|_{p} \geq\left|\delta / \delta^{\prime}\right|_{p}
$$

which is a contradiction.

We may assume $C_{p}$ appears in the same rows of $B_{p}$ for each $\mathfrak{p}$. We denote the submatrix of $B$ in these $m$ rows by $C$. The other rows of $B$ may be represented as linear combinations of the $m$ rows of $C$. By Cramer's rule, the coefficients in these combinations will be of the form $\operatorname{det} C^{\prime} / \operatorname{det} C$ where $C^{\prime}$ is some submatrix of $B$. But, by the choice of $C$, det $C^{\prime} / \operatorname{det} C \leq 1$. Hence, because all $p \in S$ are nonarchimedean, the inequalities (5) hold if and only if the inequalities hold for the rows of $C$. Hence, $E^{\prime}=C^{-1} E$ and therefore

$$
\mu\left(E^{\prime}\right)=\mu\left(C^{-1} E\right)=\left(\bmod C^{-1}\right) \mu(E)=\left|\operatorname{det} C^{-1}\right|_{S}=\left|\epsilon^{r} \delta^{-1}\right|_{S} .
$$

This proves the proposition.

A theorem similar to Proposition 4 may be found in [6].

Suppose $L$ has the form

$$
L_{i}(z)=\sum_{j=1}^{s} a_{i j} z_{j}-z_{s+i}, \quad 1 \leq i \leq r,
$$

with $m=r+s$. Then $\delta=\delta^{\prime}$ and both are ideles.

We now compute the polar lattice of $\Lambda_{L, \epsilon}$ when $L$ has the special form (6). Let $M$ be the transposed system

$$
M_{j}(w)=w_{j}+\sum_{i=1}^{r} a_{i j} w_{i+s}, \quad 1 \leq j \leq s,
$$

so that

$$
z \cdot w=-\sum_{i=1}^{r} L_{i}(z) w_{i+s}+\sum_{j=1}^{s} M_{j}(w) z_{j}
$$

Define $a_{L}=a$ to be the integral ideal of $k$ consisting of all $a$ in $D$ for which $a a_{i j} \leq 1,1 \leq i \leq r, 1 \leq j \leq s$. Also, define $\mathfrak{b}=\mathfrak{b}_{\epsilon}$ to be the ideal $\mathfrak{b}_{\epsilon}=\Pi_{p \in S} \mathfrak{b}^{\nu_{p}(\epsilon)}$; so $a \in D$ is such that $a \leq \epsilon$ if and only if $a \in b$. We now prove bolds.

Proposition 5. $b_{\epsilon} a_{L} \Lambda_{L, \epsilon}^{-1} \subseteq \Lambda_{M, \epsilon}$ If all the $a_{i j}$ satisfy $a_{i j} \leq 1$, then equality 
Proof. Let $e_{i}$ be the m-tuple with 1 in the $i$ th position and 0 elsewhere. It is clear $\mathrm{Bae}_{i} \subseteq \Lambda_{L}$. So $\left(\mathrm{bae}_{i}\right) \cdot \Lambda_{L}^{-1} \subseteq \mathrm{D}$, and therefore $\mathrm{ba} \Lambda_{L}^{-1} \subseteq \mathrm{D}^{m}$. Since $k$ is dense in $k_{S}$, we can replace the $a_{i j}$ by elements of $k$ and still get the same lattices $\Lambda_{L, \epsilon}, \Lambda_{M, \epsilon^{*}}$ So assume $a_{i j} \in k$ and set

$$
a_{j}=\left(0, \ldots, 0,1,0, \ldots, 0, a_{1 j}, \cdots, a_{r j}\right) \in k^{m}
$$

where the 1 is in the $j$ th position. Because $a a_{j} \in 0^{m}$ and $L_{i}\left(a a_{j}\right)=0$, then $a a_{i} \subseteq \Lambda_{L}$, so $\left(a a_{j}\right) \cdot \Lambda_{L}^{-1} \subseteq D_{\text {. By }}(7)$, with $w \in a b \Lambda_{L}^{-1}$ and $z \in a a_{j}$, we get

$$
M_{j}\left(a b \Lambda_{L}^{-1}\right) a=\left(a b \Lambda_{L}^{-1}\right) \cdot a a_{j}=a b\left(\Lambda_{L}^{-1} \cdot a a_{j}\right) \subseteq a b
$$

so canceling the $a^{\prime}$ s we have $a b \Lambda_{L}^{-1} \subseteq \Lambda_{M}$, as desired.

Now assume $a_{i j} \leq 1$ for all $i$ and $j$. So $a=0$, and we can assume $a_{i j} \in D_{0}$ Let $w \in \Lambda_{M}$ and $z \in \Lambda_{L}$. Then $M_{j}(w) \in \mathfrak{b}$ and $L_{i}(z) \in \mathfrak{b}$. So, by equation (7), we see that $z \cdot w \in \mathfrak{b}$, and therefore $z \cdot\left(\mathfrak{b}^{-1} w\right) \in \mathbb{D}$. This shows that $\mathfrak{b}^{-1} \Lambda_{M} \subseteq \Lambda_{L}^{-1}$, as desired.

It is easy to produce an example to show that equality does not in general hold in Proposition 5.

5. Proof of the theorem. Let $\epsilon$ be an idele with $\psi(0) \geq \epsilon \geq \psi(N)$ and satisfying

( $\left.v^{\prime}\right)|\epsilon|_{D_{1}}|\epsilon|_{p_{2}}^{-1} \leq C$ for all infinite primes $p_{1}, p_{2}$ where $C$ is the constant of condition ( $v$ ). Set $l_{N}=N / F(N)$ and note $1 \leq l_{N} \leq N$ if $N$ is sufficiently large. We first find an estimate of the number $a(N, \epsilon)$ of solutions $x \in D^{s}$, and $y \in D^{r}$ of the inequalities

$$
L_{i}(x)-y_{i} \leq \epsilon, \quad N-l_{N} \leq|x| N .
$$

Define systems $\bar{L}$ and $\bar{M}$ by the formulas

$$
\begin{gathered}
\bar{L}_{i}(z)= \begin{cases}z_{i} & \text { for } 1 \leq i \leq s, \\
\frac{-l_{N}}{\epsilon^{\infty}}\left(\sum_{j=1}^{s} a_{i-s j}^{\infty} z_{j}-z_{i}\right) & \text { for } s+1 \leq i \leq m,\end{cases} \\
\bar{M}_{j}(z)= \begin{cases}z_{j}+\sum_{i=1}^{r} a_{i j}^{\infty} z_{s+i} & \text { for } i \leq j \leq s, \\
\frac{\epsilon}{l_{N}} z_{j} & \text { for } s+1 \leq j \leq m,\end{cases}
\end{gathered}
$$

where for $a \in k_{S}$, as usual, $a^{\infty}$ denotes the $k_{\infty}$ component of $a$. Note, we are assuming real numbers such as $l_{N}$ are embedded along the diagonal in $k_{\infty}$. Let 
$L^{0}$ be as in (1) and define $M^{0}$ by

$$
M_{j}^{0}(z)=z_{j}+\sum_{i=1}^{r} a_{i j}^{0} z_{s+i}, \quad 1 \leq j \leq s .
$$

In Example 2 of $\$ 4$ we used $L^{0}$ and $\epsilon^{0}$ to define an D-lattice $\Lambda_{L 0}=\Lambda_{L 0, \epsilon^{0}}$ with determinant

$$
\operatorname{det} \Lambda_{L^{0}}=2^{-m R}{ }^{-m}|d|^{m / 2}\left|\epsilon^{-r} \delta\right|_{S_{0}}
$$

Then, by Example 1 of $\$ 4, \Lambda=\bar{L}\left(\Lambda_{L 0}\right)$ is an D-lattice with determinant

$$
\operatorname{det} \Lambda=\left|\left(\frac{l_{N}}{\epsilon^{\infty}}\right)^{r}\right|_{\infty} \operatorname{det} \Lambda_{L^{0}}=\frac{\gamma_{1} l_{N}^{r n}}{|\epsilon|_{S}^{r}}
$$

with $\gamma_{1}=2^{-m R 2}|d|^{m / 2}|\delta(L)|_{s_{0}}$. We see $a(N, \epsilon)$ is just the number of points of $\Lambda$ in the region $T$ of $k_{\infty}^{m}$ consisting of all $z \in k_{\infty}^{m}$ satisfying

$$
\begin{aligned}
N-l_{N} & \leq|x| \leq N, \quad x=\left(z_{1}, \cdots, z_{s}\right), \\
z_{i} & \leq l_{N}, \quad i=s+1, \cdots, m .
\end{aligned}
$$

Let $B_{b}$ be the boundary of $T$ expanded by the diameter $b$ of some fundamental parallelepiped of $\Lambda \subseteq R^{n m}$. Then, if $\mu$ is Lebesgue measure on $R^{n m}$, we see that

$$
a(N, \epsilon)=\frac{\mu T}{\operatorname{det} \Lambda}+o\left(\frac{\mu B_{b}}{\operatorname{det} \Lambda}\right) .
$$

We have

$$
\begin{aligned}
\mu T & =\left(\left(2^{R} 1_{\pi}{ }^{R}{ }^{2} N^{n}\right)^{s}-\left(2^{R}{ }_{1}{ }^{R}{ }^{2}\left(N-l_{N}\right)^{n}\right)^{s}\right)\left(2^{R}{ }_{1}{ }^{R}{ }_{2} l_{N}^{n}\right)^{r} \\
& =\left(2^{R}{ }^{1}{ }^{R}{ }^{2}\right)^{m} l_{N}^{n r} \int_{N-l_{N}}^{N} n s t^{n s-1} d t
\end{aligned}
$$

and

$$
\mu B_{b}=O\left(N^{n s-1} l_{N}^{r n} b\right)
$$

if

$$
b \ll l_{N}
$$

Using in (9) the value for $\operatorname{det} \Lambda_{\text {given in (8), we get }}$

$$
\alpha(N, \epsilon)=\gamma|\epsilon|_{S}^{r} \int_{N-l}^{N} t^{n s-1} d t+O\left(N^{n s-1}|\epsilon|_{S}^{r} b\right)
$$

provided that (10) holds, where 


$$
\gamma=n s\left(2^{R} 1_{\pi}^{R}\right)^{m} / \gamma_{1}=2^{m R_{\pi}^{m R}}{ }^{m}|\delta(L)|_{s_{0}}^{-1}|d|^{-m / 2} n s .
$$

We now find an upper bound for $b$. Let $\mu_{1}, \cdots, \mu_{m n}$ be the successive minimum of $\Lambda$ with respect to the distance function $f^{*}$ polar to the distance function $f: k_{\infty}^{m} \rightarrow \mathbf{R}_{+}, f(z)=\mid z$. It can be shown (see [3, Chapter V, Lemma 8]) there is a basis $c_{1}, \cdots, c_{n}$ of $\Lambda$ satisfying $f^{*}\left(c_{i}\right) \leq 1 / 2 n m \mu_{i}$. So, if we choose $b$ to be the diameter of the fundamental parallelepiped determined by this basis, we see that

$$
b \leq \sum\left|c_{i}\right| \ll \sum f^{*}\left(c_{i}\right) \ll \mu_{n m} .
$$

By Mahler's theorem (see [3]), if $\mu_{1}^{*}$ is the first minimum of $\Lambda^{*}$ with respect to $f$, then

$$
\mu_{1}^{*} \mu_{n m} \ll 1
$$

So, we can find an upper bound for $\mu_{n m}$ and hence for $b$ by finding a lower bound for $\mu_{1}^{*}$. This is where we use the type condition.

If $a=a_{L 0}, b=b_{\epsilon}$, and $\Lambda_{M^{0}}=\Lambda_{M^{0}, \epsilon^{0}}$ are defined as in $\$ 4$, we know

$$
\Lambda_{L^{0}}^{*} \subseteq c^{-1} \Lambda_{M^{0}}
$$

where $C=\operatorname{Dba} \subseteq \mathrm{D}$. Now $\bar{M}$ and $\bar{L}$ are such that

$$
\bar{M}\left(z^{\prime}\right) \cdot L\left(z^{\prime \prime}\right)=z^{\prime} \cdot z^{\prime \prime}
$$

so, as in Example 1 of $\S 4$, the lattices $\Lambda$ and $\Lambda^{*}=\bar{M}\left(\Lambda_{L 0}^{*}\right)$ are polar. Define

$$
\bar{\Lambda}=\bar{M}\left(c^{-1} \Lambda_{M} 0\right.
$$

Then, by (13), $\Lambda^{*} \subseteq \bar{\Lambda}$. So, if $\bar{\mu}_{1}$ is the first minimum of $\bar{\Lambda}$ with respect to $f$, then $\bar{\mu}_{1} \leq \mu_{1}^{*}$. Hence, we will find a lower bound for $\bar{\mu}_{1}$.

Choose $z^{\prime} \in c^{-1} \Lambda_{M^{0}}$ such that $f\left(z^{\prime}\right)=|z|=\ddot{\mu}_{1}$. By a simple application of Minkowski's theorem, there is $c \in C$ such that

$$
\left\lceil\leq\left(2^{R} \pi^{-R}{ }^{-R}|d|^{1 / 2} \text { Norm } c\right)^{1 / n}\right. \text {. }
$$

By the definition of $\mathfrak{b}$ given in $\$ 4$, we have

$$
\text { Norm } \mathfrak{b}=\prod_{p \in S_{0}} N p^{\nu_{p}(\epsilon)}=|\epsilon| s_{0}^{-1},
$$

so $|c|<|\epsilon|_{S_{0}}^{-1 / n}$ and therefore, also, $\mid$ Norm $\left.c|\ll| \epsilon\right|_{S_{0}} ^{-1}$ where the constants implied by $\ll$ do not depend on $N$.

We have $z=c z^{\prime} \in \Lambda_{M^{0}} \subseteq D^{m}$. Hence, with this $z=(x, y)$, we have 


$$
x_{j}+\sum_{i=1}^{r} a_{i j}^{0} y_{i} \leq \epsilon^{0}, \quad 1 \leq j \leq s .
$$

From the definition of $f$ and $\bar{\Lambda}$ we see that

$$
\begin{aligned}
x_{j}+\sum_{i=1}^{r} a_{i j}^{\infty} y_{i} \leq c \bar{\mu}_{1}, & 1 \leq j \leq s, \\
y_{i} \leq \frac{l_{N}}{\epsilon} c \bar{\mu}_{1}, & 1 \leq i \leq r
\end{aligned}
$$

Hence $\max _{j}\left|x_{j}+M_{j}(y)\right|_{S} \leq \mid$ Norm $\left.c\left|\bar{\mu}_{1}^{n}\right| \epsilon\right|_{S_{0}} \ll \tilde{\mu}_{1}^{n}$. By the type condition, this implies

$$
g(\mid \bar{z})^{-1}|\bar{z}|^{-r n / s} \ll \bar{\mu}_{1}^{n}
$$

By (14) and condition $\left(\nabla^{\prime}\right)$ for $\epsilon$,

$$
\sqrt{\eta} \leq l_{N} \bar{\mu}_{1} \sqrt{C}\left|\epsilon^{-1}\right| \ll l_{N} \bar{\mu}_{1}|\epsilon|_{S_{0}}^{-1 / n}|\epsilon|_{\infty}^{-1 / n}=l_{N} \bar{\mu}_{1}|\epsilon|_{S}^{-1 / n} .
$$

We also have $|x|<l_{N} \bar{\mu}_{1}|\epsilon|^{-1 / n}$ from (14), since $\epsilon \leq \psi(0)$ implies that $c \bar{\mu}_{1} \leq$ $\left(l_{N} / \epsilon\right) c \bar{\mu}_{1}$ for large $N$. Therefore $|z| \ll l_{N} \bar{\mu}_{1}|\epsilon|_{s}^{-1 / n}$, and then by (15)

$$
|\epsilon|_{S}^{r / s} l_{N}^{-r n / s} \bar{\mu}_{1}^{-r n / s} g(\sqrt{z} \mid)^{-1} \ll \bar{\mu}_{1}^{n}
$$

Solving for $\bar{\mu}_{1}$ we get

$$
\left(|\epsilon|_{s}^{r} l_{N}^{-r n} g(|\bar{z}|)^{-s}\right)^{1 / m n} \ll \bar{\mu}_{1^{\circ}}
$$

Minkowski's convex body theorem says $\bar{\mu}_{1}^{n m} \leq 2^{n m} \operatorname{det}(\bar{\Lambda}) / V_{f}$ where $V_{f}$ is the volume of the region defined by $f(z) \leq 1$. It is easy to see (in the same way we got (8)) that

$$
\bar{\mu}_{1}^{n m} \ll \operatorname{det}(\bar{\Lambda})=\operatorname{Norm} c^{-m}\left(|\epsilon|_{\infty}^{r} l_{N}^{-r n}\right)\left(2^{-m R}{ }^{-m}|d|^{m / 2}\left|\epsilon^{-s} \delta\right|_{S_{0}}\right) \ll|\epsilon|_{S}^{r} l_{N}^{-r n}
$$

So, by our bound for $\boldsymbol{z}$, we have

$$
|z|^{m n} \ll l_{N}^{m n}|\epsilon|_{s}^{-m} \bar{\mu}_{1}^{m n} \ll l_{N}^{s n}|\epsilon|_{s}^{-s}=N^{s n} / F(N)^{s n}|\epsilon|_{S^{\circ}}^{s}
$$

From condition (iii), it is now easy to see that $\sqrt{z} \leq N^{s / r}$ if $N$ is large. Hence by (16)

$$
\left(|\epsilon|_{s}^{r} l_{N}^{-r n} g\left(N^{s / \eta}\right)^{-s}\right)^{1 / m n} \ll \bar{\mu}_{1} \leq \mu_{1}^{*}
$$

and therefore from (12) and condition (iii)

$$
b \ll \mu_{n m} \ll\left(g\left(N^{s / \eta}\right)^{s} l_{N}^{r n}|\epsilon|_{s}^{-\eta}\right)^{1 / m n} \ll l_{N} F(N)^{-1} .
$$


Now (10) is clearly satisfied, so (11) now reads

$$
\alpha(N, \epsilon)=\gamma|\epsilon|_{S}^{r} \int_{N-l_{N}}^{N} t^{n s-1} d t+O\left(N^{n s-1}|\epsilon|_{S}^{r} l_{N} F(N)^{-1}\right) .
$$

The rest of the proof follows Lang [5]. We apply formula (17) to $\epsilon=\psi(N)$ and $\epsilon=\psi\left(N-l_{N}\right)$ to get the theorem. Since $\psi$ is decreasing we see

$$
a(N, \psi(N)) \leq \lambda(N)-\lambda\left(N-l_{N}\right) \leq a\left(N, \psi\left(N-l_{N}\right)\right) .
$$

Then, by (17) with $\epsilon=\psi(N)$ and $\epsilon=\psi\left(N-l_{N}\right)$,

$$
\begin{aligned}
\lambda(N)-\lambda\left(N-l_{N}\right) & =\gamma|\psi(N)|_{S}^{r} \int_{N-l_{N}}^{N} t^{n s-1} d t \\
& \left.+d\left(\left|\psi\left(N-l_{N}\right)\right|_{S}^{r}-|\psi(N)|_{S}^{r}\right) N^{n s-1} l_{N}+\frac{\left|\psi\left(N-l_{N}\right)\right|_{S}^{r} N^{s n-1} l_{N}}{F(N)}\right) .
\end{aligned}
$$

Note, $F$ increasing implies $|\psi(t)|_{s}^{r} t^{s n}$ is also increasing. Hence so

$$
\left|\psi\left(N-l_{N}\right)\right|_{S}^{r}\left(N-l_{N}\right)^{s n} \leq|\psi(N)|_{S}^{r} N^{s n} \leq|\psi(N)|_{S}^{r}\left(\left(N-l_{N}\right)^{s n}+s n N^{s n-1} l_{N}\right),
$$

$$
\left|\psi\left(N-l_{N}\right)\right|_{S}^{r}-|\psi(N)|_{S}^{r} \leq \frac{s n N^{s n-1}|\psi(N)|_{S}^{r} l_{N}}{\left(N-l_{N}\right)^{s n}} \ll \frac{l_{N}|\psi(N)|_{S}^{r}}{N}=\frac{|\psi(N)|_{S}^{r}}{F(N)}
$$

and therefore also $\left|\psi\left(N-l_{N}\right)\right|_{S}^{r} \ll|\psi(N)|_{S}^{r}$. Using these estimates in (18) we get

(19) $\lambda(N)-\lambda\left(N-l_{N}\right)=\gamma|\psi(N)|_{S}^{r} \int_{N-l_{N}}^{N} t^{s n-1} d t+o\left(\frac{|\psi(N)|_{S}^{r} N^{s n-1} l_{N}}{F(N)}\right)$.

Now $F(t) \rightarrow \infty$. So if $N$ is large enough $N-l_{N} \geq N(1-1 / F(N)) \geq N / 2$ and therefore, because $\psi(t)$ and $1 / F(t)$ are both decreasing,

(20) $\frac{|\psi(N)|_{S}^{r} N^{s n-1} l_{N}}{F(N)} \ll \frac{|\psi(N)|_{S}^{r}\left(N-l_{N}\right)^{s n-1} l_{N}}{F(N)} \leq \int_{N-l}^{N} \frac{|\psi(t)|_{S}^{r} t^{s n-1}}{F(t)} d t$.

Also, because $\psi$ is decreasing, we get

$$
\begin{aligned}
|\psi(N)|_{S}^{r} \int_{N-l_{N}}^{N} t^{s n-1} d t= & \int_{N-l_{N}}^{N}|\psi(t)|_{S}^{r} t^{s n-1} d t \\
& +O\left(\left(\left|\psi\left(N-l_{N}\right)\right|_{S}^{r}-|\psi(N)|_{S}^{r}\right) N^{n s-1} l_{N}\right)
\end{aligned}
$$

We have already estimated the error term in this last expression. Hence (19), (20), and (21) yield 


$$
\lambda(N)-\lambda\left(N-l_{N}\right)=\gamma \int_{N-l_{N}}^{N}|\psi(t)|_{s}^{r} t^{s n-1} d t+O\left(\int_{N-l_{N}}^{N} \frac{|\psi(t)|_{s}^{r} t^{s n-1}}{F(t)} d t\right)
$$

Equation (4) now follows by induction.

6. A metric theorem. We put a measure on the space of all systems $L$ of $r$ linear forms in $s$ variables by identifying the form $L$ with an rs-tuple in $k_{S}^{r s}$ made up of the coefficients of $L$. We will determine a type for almost all systems $L$. For simplicity, we restrict ourselves to the case when $S \supseteq P_{\infty}$. As preparation we state the following adele version of the convergence theorem:

Proposition 6. Let $\epsilon: \mathbf{R}_{+} \rightarrow k_{S}^{*}$. If $\Sigma_{x \epsilon_{0} s}|\epsilon(|x|)|_{S}^{r}<\infty$ then, for almost all systems $L$, there are only finitely many solutions $x \in D^{s}, y \in D^{r}$ of

$$
L_{i}(x)-y_{i} \leq \epsilon(\sqrt{x}), \quad 1 \leq i \leq r .
$$

This is the easy part of the Khinchin metric theorem; the other part asserts that, if the above sum diverges, then, under certain conditions, for almost all systems $L(22)$ will have infinitely many solutions. A proof of this theorem for the adeles, in the case $s=1$, may be found in [2].

If $k=\mathbf{Q}$ and $S=P_{\infty}$, the above proposition gives a type for almost all systems $L$. However, in the general case, type is defined in terms of an inequality on the volume $\mid I_{s}$ and not by simultaneous inequalities such as in (22), so the proposition does not apply directly. By modifying the proof of a theorem in [4, p. 96] we can get what we need, if the set of primes $S$ is finite.

Proposition 7. Let $S \supseteq P_{\infty}$ be a finite set of primes, and let $\epsilon: \mathbf{R}_{+} \rightarrow \mathbf{R}_{+}$If

$$
\epsilon(t)<1 \text { and } \int_{1}^{\infty} t^{n m-1} \epsilon(t)^{r(1-\eta)} d t<\infty, \quad 1>\eta>0 \text {, }
$$

then for almost all $L$, there are only finitely many $x \in D^{s}, y \in D^{r}$ satisfying

$$
\max _{i}\left|L_{i}(x)-y_{i}\right|_{s} \leq \epsilon(\sqrt{z}), \quad z=(x, y) .
$$

Proof. It is easy to see, if we replace (22) by

$$
\inf \left\{1, L_{i}(x)-y_{i}\right\} \leq \epsilon(\sqrt{z}), \quad z=(x, y), 1 \leq i \leq r,
$$

then the proof of Proposition 6 shows that for almost all systems $L$ the inequalities (24) have only a finite number of solutions when $\int_{1}^{\infty} t^{n m-1}|\epsilon(t)|_{S}^{r} d t<\infty$ (the $\epsilon$ in (24) is as in Proposition 6, i.e., $\epsilon: \mathbf{R}_{+} \rightarrow k_{S}^{*}$ ).

For the proof of Proposition 7, we assume, for the sake of simplicity, that $r=$ 1. Let $F$ be the set of all $L$ for which $(23)$ has infinitely many solutions. Suppose (23) holds for $z=(x, y)$. If we put

$$
\inf \left\{1,\left|L_{1}(x)-y_{1}\right|_{p}\right\}=\epsilon(\mid \bar{z})^{\tau_{p}(z)},
$$


then $\tau_{p}=\tau_{p}(z) \geq 0$ and $\Sigma_{p} \tau_{p} \geq 1$. Let $v$ be the number of elements in $S$, and choose a positive integer $A$ so large that $v / A<\eta$. We have $A \leq\left[\Sigma_{p} A \tau_{p}\right] \leq$ $\Sigma_{p}\left[A \tau_{p}\right]+v$; and therefore, if $B=A-v>0$, then $B \leq \Sigma_{p}\left[A \tau_{p}(z)\right]$. So there exists $b_{p}=b_{p}(z)$ such that $b_{p}$ is an integer and

$$
0 \leq b_{p} \leq\left[A \tau_{p}(z)\right] \leq A \tau_{p}(z), \quad \sum_{p} b_{p}=B .
$$

There are only a finite number of possibilities for each $b_{p}$. So, if $L \in F$, we may assume, for each $p \in S, b_{p}=b_{p}(z)$ takes on the same value for infinitely many solutions $z=(x, y)$ of (23); i.e., we may assume $b_{p}$ takes on a value depending only on $L$ and not on $z$. By (26), if we set $l_{p}=b_{p} / A$, then

$$
0 \leq l_{p} \leq \tau_{p}, \quad \sum_{p} l_{p}=B / A=(A-v) / A>1-\eta .
$$

Then (25) implies there are infinitely many solutions of

$$
\inf \left\{1,\left|L_{1}-y_{1}\right|_{p}\right\} \leq \epsilon(|z|)^{l_{p}} \text {. }
$$

Now $\Pi_{p} \epsilon(t)^{l_{p}} \leq \epsilon(t)^{1-\eta}$. Therefore, since $\int_{1}^{\infty} t^{n m-1} \epsilon(t)^{1-\eta} d t$ converges, we see that the set $E(b), b=\left(b_{p}\right)_{p \in S}$, for which (27) has infinitely many solutions, has measure zero. But $F \subseteq \bigcup_{E(b)}$ where the union is over all tuples $b=\left(b_{p}\right)$ with $b_{p} \geq 0$ and $\Sigma_{p} b_{p}=B$. So the measure of $F$ is also zero. This proves Proposition 7.

If we apply Propositon 7 to the transposed system $M$ of $s$ forms in $r$ variables, we find that by taking $g(t)$ so that

$$
\int_{1}^{\infty} \frac{t^{n m-1}}{\left(g(t) t^{m / s}\right)^{s(1-\eta)}} d t \text { converges, }
$$

then almost all $L$ have type $\leq g$. So, for a $g$ satisfying (28) and a $\psi$ satisfying conditions (ii)-(v), we have that formula (4) holds for almost all $L$.

It may be possible that Proposition 7 can be refined, and therefore a better metric theorem would result. For example, in the case $k=\mathbf{Q}, S=P_{\infty}$, almost all systems have type $\leq \log ^{1+\eta} t$, while Proposition 7 can never give a type any better than $O\left(t^{\alpha}\right)$. Also, in the case $k=\mathbf{Q}$ and $S$ consists of one $p$-adic prime, one can show almost all systems $L$ have type $\leq \log ^{1+\eta} t$ (see the Khinchin metric theorem in [6] where it is shown that almost all $p$-adic systems

$$
\left|L_{i}(x)-y_{i}\right|_{p} \leq \epsilon(t), \quad t=\max _{i, j}\left\{\left|x_{j}\right|,\left|y_{i}\right|\right\}
$$

have only a finite number of solutions, if $t \epsilon(t)$ is decreasing and $\left.\Sigma t^{m-1} \epsilon(t)^{r}<\infty\right)$. However, if $S$ contains more than one infinite prime it seems unlikely the integral in Proposition 7 can be improved to anything better than 


$$
\int_{1}^{\infty} t^{n s-1} \epsilon(t)^{r} \log \epsilon(t)^{-1} d t
$$

since, for example, the measure of the set

$$
\left\{(a, b) \in \mathbf{R}^{2}: \inf \{1,|a|\} \inf \{1,|b|\} \leq \epsilon\right\}
$$

is of the form $2 \epsilon\left(1+2 \log \epsilon^{-1}\right)$.

In the case $s<r$ our theorem will still hold if we replace the definition of type with the following definition of $\psi$-type:

Definition. Let $g: \mathbf{R}_{+} \rightarrow \mathbf{R}_{+}$be an increasing function, and let $\psi: \mathbf{R}_{+} \rightarrow k_{S}^{*}$. Define $\epsilon(t)$ by the formulas

$$
\begin{aligned}
& \epsilon_{p}(t)=\psi_{p}(t) \text { for } p \in S_{0}, \\
& \epsilon_{p}(t)=\left(g(t) t^{m / s}|\psi(t)|_{S_{0}}\right)^{-1 / n} \text { for } \mathfrak{p} \in P_{\infty},
\end{aligned}
$$

Then we say the system $L$ has $\psi$-type $\leq g$, if $M_{j}(y)-x_{j} \leq \epsilon(\sqrt{y}), 1 \leq j \leq s$, has only finitely many solutions $y \in D^{r}$ and $x \in D^{s}$.

In this case we may apply the Khinchin convergence theorem (Proposition 6 ) directly to obtain the following metric corollary to the type theorem:

Proposition 8. Assume $s<r$. If $\int_{1}^{\infty} g(t)^{-s} t^{-1} d t$ converges and conditions (ii) through ( $v$ ) of the type theorem bold, then

for almost all systems $L$.

$$
\lambda(N) \sim \gamma \int_{1}^{N} t^{s n-1}|\psi(t)|_{s}^{r} d t
$$

\section{REFERENCES}

1. W. W. Adams, Simultaneous asymptotic diophantine approximations, Mathematika 14 (1967), 173-180. MR 36 \#3730.

2. D. G. Cantor, On the elementary theory of diophantine approximation over the ring of adeles. I, Illinois J. Math. 9 (1965), 677-700. MR 32 \#5592.

3. J. W. S. Cassels, An introduction to the geometry of numbers, Di e Grundlehren der math. Wissenschaften in Einzeldarstellungen mit besonderer Berücksichtigung der Anwendungsgebiete, Band 99, Springer-Verlag, Berlin, 1959. MR 28 \#1175.

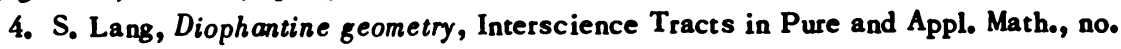
11, Interscience, New York, 1962. MR 26\#119.

5. —_, Introduction to diophantine approximations, Addison-Wesley, Reading, Mass., 1966. MR 35 \#129.

6. É. Lutz, Sur les approximations diophantiennes linéaires p-adiques, Actualités Sci. Indust., no. 1224, Hermann, Paris, 1955. MR 16, 1003.

7. K. Rogers and H. P. F. Swinnerton-Dyer, The geometry of numbers over algebraic number fields, Trans. Amer. Math. Soc. 88 (1958), 227-242. MR 20 \#1666.

8. M. Sweet, $A$ theorem in diophantine approximations, J. Number Theory 5 (1973), $245-251$. 21228

DEPARTMENT OF MATHEMATICS, UNIVERSITY OF MARYLAND, BALTIMORE, MARYLAND 